\title{
In situ epicatechin-loaded hydrogel implants for local drug delivery to spinal column for effective management of post-traumatic spinal injuries
}

\author{
Jun Lu*, You-ting Ju, Shou-lin Chen, Jun-ying Cai, Guo-hai Xu and Yan-hui Hu \\ Department of Anesthesiology, The Second Affiliated Hospital to Nanchang University, Nanchang, Jiangxi, China
}

${ }^{*}$ For correspondence: Email: lujun2611@hotmail.com

Received: 18 January 2016

Revised accepted: 6 June 2016

\begin{abstract}
Purpose: To prepare hydrogels loaded with epicatechin, a strong antioxidant, anti-inflammatory, and neuroprotective tea flavonoid, and characterise them in situ as a vehicle for prolonged and safer drug delivery in patients with post-traumatic spinal cord injury.

Methods: Five in situ gel formulations were prepared using chitosan and evaluated in terms of their visual appearance, clarity, $\mathrm{pH}$, viscosity, and in vitro drug release. In vivo anti-inflammatory activity was determined and compared with $2 \%$ piroxicam gel as standard. Motor function activity in a rat model of spinal injury was examined comparatively with i.v. methylprednisolone as standard.

Results: The N-methyl pyrrolidone solution (containing $1 \% \mathrm{w} / \mathrm{w}$ epicatechin with 2 to $10 \% \mathrm{w} / \mathrm{w}$ chitosan) of the in situ gel formulation had a uniform $\mathrm{pH}$ in the range of $4.01 \pm 0.12$ to $4.27 \pm 0.02$. High and uniform drug loading, ranging from $94.48 \pm 1.28$ to $98.08 \pm 1.24 \%$, and good in vitro drug release (79.48 \pm 2.84 to $96.48 \pm 1.02 \%$ after 7 days) were achieved. The in situ gel prepared from $1 \%$ epicatechin and $2 \%$ chitosan (E5) showed the greatest in vivo anti-inflammatory activity $(60.58 \%$ inhibition of paw oedema in standard carrageenan-induced hind rat paw oedema model, compared with $48.08 \%$ for the standard). The gels showed significant therapeutic effectiveness against post-traumainduced spinal injury in rats. E5 elicited maximum motor activity (horizontal bar test) in the spinal injury rat model; the rats that received E5 treatment produced an activity score of $3.62 \pm 0.02$ at the end of 7 days, compared with $5.0 \pm 0.20$ following treatment with the standard.

Conclusion: In situ epicatechin-loaded gel exhibits significant neuroprotective and anti-inflammatory effects, and therefore can potentially be used for prolonged and safe drug delivery in patients with traumatic spinal cord injury.
\end{abstract}

Keywords: Epicatechin, In situ gel, Chitosan, Spinal injury, Post-traumatic, Motor activity, Antiinflammatory

Tropical Journal of Pharmaceutical Research is indexed by Science Citation Index (SciSearch), Scopus, International Pharmaceutical Abstract, Chemical Abstracts, Embase, Index Copernicus, EBSCO, African Index Medicus, JournalSeek, Journal Citation Reports/Science Edition, Directory of Open Access Journals (DOAJ), African Journal Online, Bioline International, Open-J-Gate and Pharmacy Abstracts

\section{INTRODUCTION}

Flavonoids are the largest group of bioactive polyphenols found in nature. Green and black teas are the richest sources of polyphenols, particularly flavanols and flavonols, which comprise $30 \%$ of the dry weight of the fresh leaf. These dietary flavonoids and nutraceutical compounds-particularly epicatechin (EpC), catechin, and (-)-epigallocatechin-3-gallate (EGCG), which are the chief bioactive constituents-have a broad spectrum of activity. Green tea polyphenols, including EpC, reportedly exert antioxidant, anti-inflammatory, anticarcinogenic, antiviral, anti-arthritic, antibacterial, antiangiogenic, neuroprotective, and anti- 
hyperlipidemic effects. These flavones also induce apoptosis and cell cycle arrest in a wide array of cell lines, and protect against cerebral ischaemia [1,2].

Among the various polyphenols, EpC reportedly exhibits a wide range of biological activities, such as antioxidant, antibacterial, anti-carcinogenic, and anti-tumour effects $[3,4]$. The neuroprotective effects induced by EpC and other various green tea extracts include attenuated ischaemic brain injury and inhibition of excitotoxicity and are evidenced by improved cognitive performance and memory retention; these effects may be utilised in patients with spinal cord injury (SCI) [5-8]. Trauma-induced $\mathrm{SCl}$ is one of the most challenging conditions to treat. The primary tissue injury leads to a cascade of adverse events, such as inflammation and apoptosis, which result in neuropathic pain, inflammation, and reversible and/or irreversible damage to the nervous system, known as secondary injury [9-12].

The present work aimed to develop in situ EpCloaded hydrogels for localised drug delivery to the SC with the advantages of a reduced frequency of dosage, prolonged drug release, and improved patient compliance for the management of SCl. The prepared gels were characterised in terms of their the physicochemical properties, in vitro drug release, in vivo anti-inflammatory activity, and in vivo motor function activity.

\section{EXPERIMENTAL}

\section{Materials}

EpC and chitosan (75\% deacylated, Ch) were obtained from Sigma-Aldrich, US. Other chemicals used were of analytical grade.

\section{Preparation of in situ gels loaded with EpC}

A total of five formulations of $\mathrm{pH}$-responsive in situ gels loaded with EpC were prepared using chitosan (Table 1).

Table 1: Composition of epicatechin (EpC)-loaded in situ gels*

\begin{tabular}{lcc}
\hline $\begin{array}{l}\text { In situ } \\
\text { gel }\end{array}$ & $\begin{array}{c}\text { EpC } \\
(\% \text { w/w })\end{array}$ & $\begin{array}{c}\text { Chitosan } \\
(\% \text { w/w })\end{array}$ \\
\hline E1 & 1 & 10 \\
E2 & 1 & 8 \\
E3 & 1 & 6 \\
E4 & 1 & 4 \\
E5 & 1 & 2 \\
\hline * N-methyl-2-pyrrolidone & $($ NMP) was used as the \\
solvent & &
\end{tabular}

The polymeric solutions were prepared in NMP. EpC was gently dispersed in the solution with continuous stirring at $200 \mathrm{rpm}$. The $\mathrm{pH}$ of the resulting solution was maintained at 4.0. The solution thus obtained was degassed, followed by membrane filtration sterilisation. The prepared sterile in situ gels were stored in a sterile area in a vacuum desiccator until further use.

\section{Physicochemical characterisation}

Clarity was determined by visual inspection against a black and white background. The viscosity of the in situ gelling solution in the presence or absence of simulated biological fluid (SBF) was determined using a Brookfield viscometer. The $\mathrm{pH}$ was determined using a digital $\mathrm{pH}$ meter.

To determine the drug content of each formulation, each formulation $(5 \mathrm{~mL}$ removed after vigorous shaking) was mixed with $100 \mathrm{~mL}$ of methanol and continuously stirred at $300 \mathrm{rpm}$ for $1 \mathrm{~h}$. The resulting solution was centrifuged at $100 \mathrm{rpm}$ for $10 \mathrm{~min}$. The clear supernatant was then analysed spectrophotometrically to determine the drug content.

\section{In vitro drug release studies}

An in vitro drug release study was performed using the method reported by Che et al [13]. The formulations were first brought to a gelled state by mixing $5 \mathrm{~mL}$ of the each formulation with 15 $\mathrm{mL}$ of phosphate buffer $(\mathrm{pH} 7.4)$. This pregelled formulation was stored in a vial containing phosphate buffer $(20 \mathrm{~mL}, \mathrm{pH}$ 7.4) on a shaking water bath $\left(37 \pm 1{ }^{\circ} \mathrm{C}\right.$; 50 oscillations per min). Samples (1 $\mathrm{mL}$ each) were removed at predetermined time intervals for 1 week; at each sampling time point an equal volume of prewarmed fresh buffer was replaced in the vial. The samples were analysed by ultraviolet (UV) spectrophotometry to determine the drug release at each time point $(n=3)$.

\section{Animals}

Healthy male Wistar rats $(150-220 \mathrm{~g})$ were used in this study. The rats were kept in cages and housed under standard light and temperature conditions. They were allowed free access to drinking water and a standard diet. The animal study protocols were approved by the Animal Care and Use Committee of Nanchang University, Nanchang (approval no. 2015/02b13). The studies were carried out in compliance with Directive 2010/63/EU on the handling of animals used for scientific purposes [14]. 


\section{In vivo anti-inflammatory studies}

The in vivo anti-inflammatory activity of the formulations was evaluated using the standard carrageenan-induced rat hind paw oedema model. Rats were divided into 6 groups of 6 rats each. The control group (Group I) rats were untreated. For Groups II to $\mathrm{V}$, the in situ gel formulations were injected $(20 \mathrm{mg} / \mathrm{kg}$ body weight; intra venous) into the left hind paw of the rats. Group VI received the $2 \%$ piroxicam gel (standard). Paw oedema was induced $30 \mathrm{~min}$ later by injecting $0.1 \mathrm{~mL}$ of a $1 \% \mathrm{w} / \mathrm{v}$ aqueous suspension of carrageenan into the left paws of all rats. The hind paw volume was measured immediately $(0 \mathrm{~h})$ and at predetermined time intervals using a plethysmometer, and was expressed as percent oedema relative to the initial hind paw volume. The percent inhibition of oedema was considered to reflect the antiinflammatory activity.

\section{In vivo motor function activity}

The rats were divided into 7 groups (normal control, negative control, standard, and 4 test groups) of 6 rats each (total, 42 rats). An in vivo motor function activity study was performed as reported by Che et al [13]. With the exception of the animals in the normal control group, all animals were subjected to laminectomy (to induce acute spinal injury) after administration of thiopental sodium $(40 \mathrm{mg} / \mathrm{kg})$. Specifically, extradural $40 \mathrm{~g}$ force clip compression was performed for $20 \mathrm{~s}$ at around T6 (until motor nerve activity is lost). The control group was administered the blank (non-medicated) gel. The test group received the in situ gels $(50 \mathrm{mg} / \mathrm{kg}$ body weight; intrathecally [i.t.]) at a shaved site closest to the injury. The standard group received intravenous (i.v.) methylprednisolone (30 mg/kg body weight) at $30 \mathrm{~min}$ post-injury to allow for drug release. This daily treatment was repeated for 1 week. At the end of 7 days, motor function activity was assessed using a horizontal bar experiment. The time spent by rats on the horizontal bar was determined, and scoring was performed as follows. Activity scores were assigned according to the time spent by each rat on the horizontal bar $(1-5 \mathrm{~s}=1 ; 6-10 \mathrm{~s}=2$; $11-$ $20 \mathrm{~s}=3 ; 21-30 \mathrm{~s}=4$; >30 $\mathrm{s}=5$; if bar support touched with forepaw $=5$ for $2 \mathrm{~mm}$ bar.

\section{Statistical analysis}

The results are expressed as means \pm standard deviation (SD). Statistical analysis was performed using Origin 9 software (USA) and Student's t-tests. A value of $p<0.05$ was considered to indicate statistical significance.

\section{RESULTS}

The gelling capacity of the polymer with SBF was determined, and suitable ratios for maximum gelling were adopted for the formulations.

\section{Physicochemical evaluation}

Addition of SBF to the in situ gelling solutions, which were colourless and clear when inspected visually, resulted in formation of a viscous gel mass. The in situ gel formulation solution showed a uniform $\mathrm{pH}$ in the range of $4.01 \pm 0.12$ to $4.27 \pm$ 0.02 (Table 2).

The formulation showed high and uniform drug loading, ranging from $94.48 \pm 1.28$ to $98.08 \pm$ $1.24 \%$. The viscosity of the formulations was found to increase 5- to 7-fold (Table 2) after gelling. The formulations showed a shearthinning property (Table 2), as has been reported previously.

\section{In vitro drug release studies}

In vitro drug release studies were performed in phosphate buffer at $\mathrm{pH} 7.4$ to simulate the $\mathrm{pH}$ of biological fluid. The formulations showed drug release proportions ranging from $79.48 \pm 2.02$ to $96.48 \pm 1.05 \%$ after 7 days (Fig 1).

Table 2: Physicochemical characterisation of in situ gels loaded with EpC

\begin{tabular}{lcccc}
\hline Code & pH & $\begin{array}{c}\text { Drug } \\
\text { content } \\
(\%)\end{array}$ & $\begin{array}{c}\text { Viscosity of } \\
\text { non-gelled } \\
\text { formulation } \\
\text { (cps) }\end{array}$ & $\begin{array}{c}\text { Viscosity of } \\
\text { pre-gelled } \\
\text { formulation } \\
\text { (cps) }\end{array}$ \\
\hline E1 & $4.01 \pm 0.12$ & $95.00 \pm 2.09$ & 5.02 & 27.08 \\
E2 & $4.12 \pm 0.02$ & $98.04 \pm 1.46$ & 4.84 & 26.07 \\
E3 & $4.24 \pm 0.01$ & $97.24 \pm 2.22$ & 3.80 & 23.05 \\
E4 & $4.26 \pm 0.02$ & $94.48 \pm 1.28$ & 3.02 & 21.04 \\
E5 & $4.27 \pm 0.02$ & $98.08 \pm 1.24$ & 2.99 & 19.84 \\
\hline
\end{tabular}




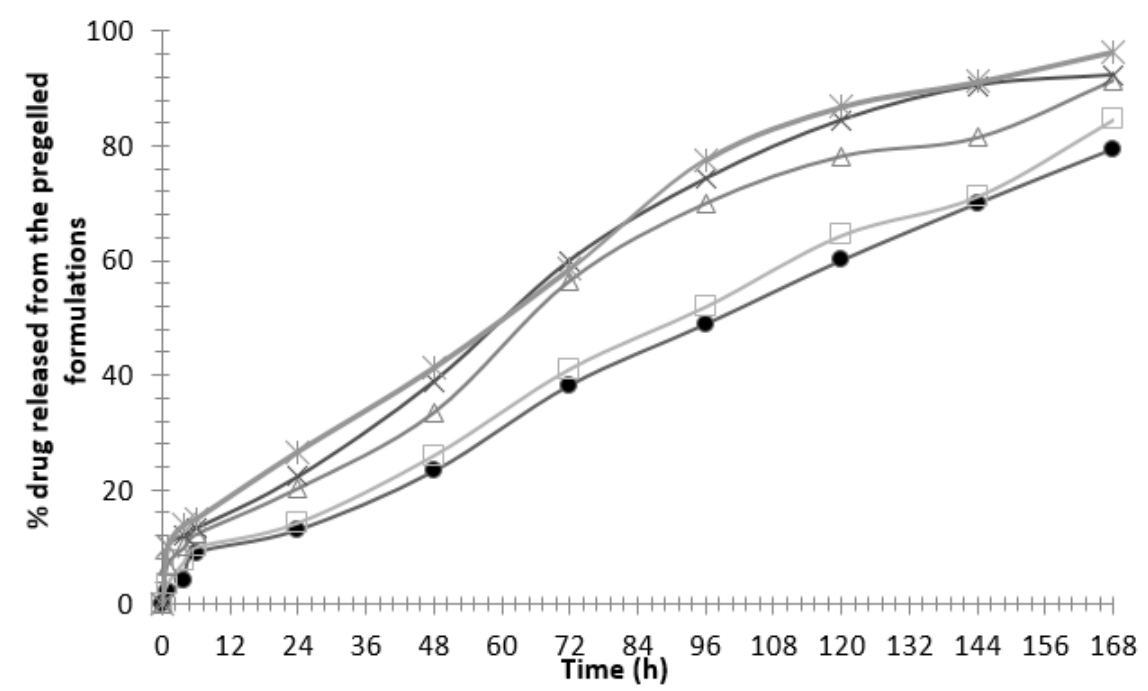

Fig. 1: In vitro epicatechin $(\mathrm{EpC})$ release from in situ gels in phosphate buffer, pH $7.4\left(-\bullet-E 1 ;-\square-\mathrm{E} 2 ;-\Delta-\mathrm{E} 3 ;-\mathbf{x}_{-}\right.$ E4; and --- E5)

\section{In vivo anti-inflammatory activity}

Inhibition of carrageenan-induced paw oedema was taken as evidence of anti-inflammatory activity (Table 3 ).

Table 3: Anti-inflammatory activity of in situ gels loaded with EpC

\begin{tabular}{lc}
\hline $\begin{array}{l}\text { Animal } \\
\text { group }\end{array}$ & $\begin{array}{c}\text { Inhibition of } \\
\text { oedema (\%) }\end{array}$ \\
\hline Control
\end{tabular}

${ }^{a} n=6 ;{ }^{b} \%$ inhibition of paw oedema $12 h$ after carrageenan injection against the paw volume of the control group $(1.04 \pm 0.10 \mathrm{~mL}) ;{ }^{c} p<0.05 \mathrm{vs}$. control
The reduction in the initial volume of paw oedema over time due to the effect of the standard drug or test formulation was assessed. The percent inhibition of paw oedema ranged from 52.88 to $60.58 \%$, compared with $48.08 \%$ for the standard.

\section{In vivo motor function activity}

The motor nerve activity elicited by the formulations was assessed using a rat model of post-traumatic SCl. After treatment for 7 days, the activity scores of the rodents treated with the in situ gels were $3.56,3.4,3.5,3.6$, and 3.62 for E1, E2, E3, E4, and E5, respectively. E5 elicited the optimum motor nerve activity in rats with traumatic SCl (Fig 2).

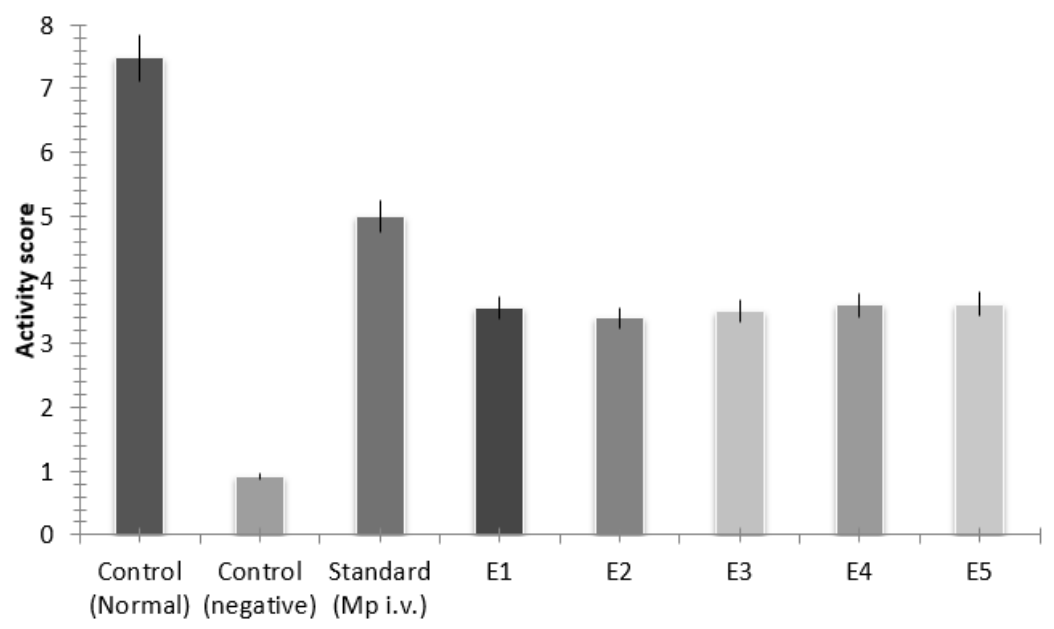

Fig 2: In vivo motor function activity elicited by in situ gels loaded with EpC in a rat model of spinal injury after 1 week of treatment 


\section{DISCUSSION}

Each year, several million people suffer from serious traumatic peripheral nerve injuries globally. If the damaged nerves cannot be restored, loss of muscle function, impaired sensation, and painful neuropathies can result. Acute steroids have been reported to be an effective first-line therapy in traumatic $\mathrm{SCl}$; indeed, a high dose of methylprednisolone given immediately after traumatic $\mathrm{SCl}$ is an accepted standard treatment [15]. Despite several reports of the effectiveness of i.v. methylprednisolone in traumatic SCl-induced nerve damage, it cannot cross the blood-spinal cord barrier. This results in the need for frequent administration of large doses of methylprednisolone.

Biodegradable polymers in the form of scaffolds, implants, or hydrogels are safe and effective platforms for site-specific or targeted drug delivery [16-19]. Localised drug delivery via intrathecal injections of polymeric systems in the SC likely elicits results comparable to those of conventional parenteral steroid therapy, but with a lower dose and prolonged drug release $[13,20,21]$. Injectable in situ hydrogel implants have been investigated in terms of their neuroprotective effects against $\mathrm{SCl}$. These polymeric solutions are injectable and are converted into a hydrogel (i.e., viscous matrix depot form) due to a change in temperature, $\mathrm{pH}$, ion cross-linking, or solvent removal [22-24].

In the present study, we prepared chitosanbased in situ hydrogel implants; hydrogel formation was triggered by a change in $\mathrm{pH}$ and solvent removal [25]. Immediately after contact with an aqueous environment, the NMP solvent diffuses out, resulting in polymer precipitation. This ultimately results in the formation of a porous polymer matrix, which slowly releases the drug into the biological microenvironment. High drug loading was observed with the in situ gels showed, a property desirable for drug delivery. The rheology of the prepared formulation was also satisfactory with the consistency in which it could be easily injected. The viscosity of the formulation was governed by the nature and amount of polymer present. In principle, the in situ gel should not alter the basic rheological properties of the biological fluid at the site of action. In this case, the formulation was delivered into the intrathecal fluid, which is crucial for neuroprotection. Further studies to assess the effect of the in situ formed hydrogel on the rheology of the intrathecal fluid are warranted.

The formulation with low chitosan content had better release properties. Use of a higher polymer content might have resulted in the formation of a stiffer gel, which would act as a barrier to drug diffusion. The in situ gel formulation E5 showed the highest antiinflammatory activity, $60.58 \%$ inhibition of paw oedema compared with $48.08 \%$ for the standard. This might have been due to the lower polymer concentration, which facilitated drug release. However, the $\mathrm{E} 1, \mathrm{E} 2$, and $\mathrm{E} 3$ formulations may function better over time, as these released the drug over a prolonged period of time. Moreover, all of the formulations had better results than those seen following treatment with the standard.

The horizontal bar method is a practical and easy-to-use method of assessing motor nerve activity in rats with post-traumatic $\mathrm{SCl}$. The activity score of the model rats following treatment with the formulations was $3.4-3.6$, and that following treatment with the standard was 5.0. Therefore, the motor nerve activity was greater after treatment with the EpC-loaded in situ gels than that following treatment with the standard.

\section{CONCLUSION}

Biodegradable polymers can be used in in situ EpC-loaded gels for continuous, extended drug release for a round-the-clock management of pain and inflammation in patients with SCl. The gel prepared from EpC and chitosan at a 1:4 ratio exhibited the greatest potential for prolonged post-traumatic therapy of $\mathrm{SCl}$ due to its considerable neuroprotective and antiinflammatory effects.

\section{DECLARATIONS}

\section{Conflict of Interest}

No conflict of interest associated with this work.

\section{Contribution of Authors}

The authors declare that this work was done by the authors named in this article and all liabilities pertaining to claims relating to the content of this article will be borne by them.

\section{REFERENCES}

1. Lee $H$, Bae $J H$, Lee SR. Protective effect of green tea polyphenol EGCG against neuronal damage and brain oedema after unilateral cerebral ischemia in gerbils. $J$ Neurosci Res 2004; 77(6): 892-900.

2. Simonyi $A$, Wang $Q$, Miller RL, Yusof $M$, Shelat $P B$, Sun $A Y$, Sun GY. Polyphenols in cerebral ischemia: novel 
targets for neuroprotection. Mol Neurobiol 2005; 31(1-3): 135-147.

3. Semalty A, Tanwar YS, Singh D, Rawat MSM. Phosphatidylcholine complex in improving oral drug delivery of epicatechin: preparation and characterization, J Drug Disc Development 2014; 1: 4655.

4. Yokozawa T, Rhyu DY, Cho EJ, Aoyagi K. Protective activity of (-)-epicatechin 3-O-gallate against peroxynitrite-mediated renal damage. Free Radic Res 2003; 37(5): 561-571.

5. Van Praag $H$, Lucero MJ, Yeo GW, Stecker K, Heivand $N$, Zhao C, Yip E, Afanador $M$, Schoroeter $H$, Hammerstone J, et al. Plant-derived flavanol (lepicatechin enhances angiogenesis and retention of spatial memory in mice. J Neurosci 2007; 27: 58695878.

6. Paterniti I, Genovese T, Crisafulli C, Mazzon E, Di Paola R, Galuppo M, Bramanti P, Cuzzocrea S. Treatment with green tea extract attenuates secondary inflammatory response in an experimental model of spinal cord trauma. Naunyn Schmiedebergs Arch Pharmacol 2009; 380(2): 179-192.

7. Zhao J, Fang S, Yuan Y, Guo Z, Zeng J, Guo Y, Tang P, Mei $X$. Green tea polyphenols protect spinal cord neurons against hydrogen peroxide-induced oxidative stress. Neural Regen Res 2014; 9(14): 1379-1385.

8. Nehlig $A$. The neuroprotective effects of cocoa flavanol and its influence on cognitive performance. $\mathrm{Br} J \mathrm{Clin}$ Pharmacol 2013; 75: 716-727.

9. Liu $M$, Zhang $W-D$, Zhang $M O$, Chen $X C$, Zou $Q$. Sustained release matrix tablet of ketorolac for effective management of pain and inflammation in spinal injuries. J Biomater Tissue Eng 2015; 5: 252-256.

10. Hagen EM, Rekand T, Gilhus NE, Grønning M. Traumatic spinal cord injuries-incidence, mechanisms and course. Tidsskr Nor Laegeforen 2012; 132 (7): 831-837.

11. Thuret S, Moon LD, Gage FH. Therapeutic interventions after spinal cord injury. Nat Rev Neurosci 2006; 7: 628643.

12. Schmidt CE, Leach JB. Neural tissue engineering: strategies for repair and regeneration. Annu Rev Biomed Eng 2003; 5: 293-347.
13. Che YJ, Chen L, Lv GH, Wang XB. In situ gel delivery system of methylprednisolone for post-traumatic spinal injuries. J Biomater Tissue Eng 2015; 5: 552-556.

14. European Commission [homepage on the internet]. Directive 2010/63/EU on the protection of animals used for scientific purposes [cited 2015 June 11]. Available from:

http://ec.europa.eu/environment/chemicals/lab_animals/l egislation_en.htm.

15. Bydon M, Lin J, Macki M, Gokaslan ZL, Bydon A. The current role of steroids in acute spinal cord injury. World Neurosurg 2014; 82(5): 848-854.

16. Huang $Y-C$, Huang $Y-Y$. Biomaterials and strategies for nerve regeneration. Artif Organs 2006; 30(7): 514-522.

17. Novikova $L N$, Novikov $L N$, Kellerth J-O. Biopolymers and biodegradable smart implants for tissue regeneration after spinal cord injury. Curr Opin Neurol 2003; 16: $711-$ 715.

18. Hoffman, AS. Hydrogels for biomedical applications. Ann N Y Acad Sci 2001; 944: 62-73.

19. Balakrishnan $B$, Jayakrishnan $A$. Self-cross-linking biopolymers as injectable in situ forming biodegradable scaffolds. Biomaterials 2009; 26: 3941-3951.

20. Jimenez Hamann MC, Tator CH, Shoichet MS. Injectable intrathecal delivery system for localized administration of EGF and FGF-2 to the injured rat spinal cord. Exp Neurol 2005; 194(1): 106-119.

21. Kang CE, PC Poon, CH Tator, MS Shoichet. A new paradigm for local and sustained release of therapeutic molecules to the injured spinal cord for neuroprotection and tissue repair. Tissue Eng Part A 2009; 15 (3): 595604.

22. Yu L, Ding J. Injectable hydrogels as unique biomedical materials. Chem Soc Rev 2008; 37: 1473-1481.

23. Liu SQ, Tay R, Khan M, Ee PLR, Hedrick JL, Yang YY. Synthetic hydrogels for controlled stem cell differentiation. Soft Matter 2010; 6: 67-81.

24. Jeong B, Gutowska A. Lessons from nature: stimulateresponsive polymers and their biomedical applications. Trends Biotechnol 2002; 20(7): 305-311.

25. Freier T, Montenegro R, Koh HS, Shoichet MS. Chitinbased tubes for tissue engineering in the nervous system. Biomaterials 2005; 26: 4624-4632. 